\title{
An Approach to Optimize the Departure Times of Transit Vehicles with Strict Capacity Constraints
}

\author{
Huimin Niu and Xiaopeng Tian \\ School of Traffic and Transportation, Lanzhou Jiaotong University, Lanzhou 730070, China \\ Correspondence should be addressed to Huimin Niu; hmniu@mail.lzjtu.cn
}

Received 21 October 2013; Revised 16 November 2013; Accepted 26 November 2013

Academic Editor: Wuhong Wang

Copyright ( $) 2013$ H. Niu and X. Tian. This is an open access article distributed under the Creative Commons Attribution License, which permits unrestricted use, distribution, and reproduction in any medium, provided the original work is properly cited.

\begin{abstract}
This paper focuses on an urban transit line which connects several residential areas and a workplace during the morning rush hours. The congestion is represented by some passengers who must wait for an extended duration and board the next or the third departure vehicles. This paper divides the time horizon equally into several small periods to measure the dynamic passenger demands. Under period-dependent demand conditions, a biobjective optimization model is developed to determine the departure times of transit vehicles at the start station with strict capacity constraints, in which a heuristic algorithm based on intelligent search and local improvement is designed to solve the model. The developed model can address the case in which more than two passengers arrive at a station simultaneously during one same period and calculate the number of boarded passengers. Finally, the model and algorithm have been successfully verified by a numerical example.
\end{abstract}

\section{Introduction}

Public transit vehicles shuttle on the lanes in accordance with a predetermined schedule to deliver the passengers from origins to destinations. The schedule for urban public transit systems is of predominant bridge between the service provider and the passenger users. At the same time, transit schedules and their compliance mirror the quality of public transit service provided. How to construct a practicable and effective schedule is a complicated task which requires the consideration of characteristics of passenger demands and benefit of the transit company as well as the operational regulations.

As a matter of fact, the key problem of designing a schedule for an urban public transit line is to determine the departure times of transit vehicles at the start station. This paper considers the scheduling problem for an urban transit line which connects several residential areas and a workplace during the morning rush hours. The spatial and temporal imbalances of demand distributions represent the transit congestion that occurs only at some stops or during some times. Under such conditions, some waiting passengers cannot board the current transit vehicle due to the limited capacity. A constant-headway schedule cannot satisfy effectively the time-dependent passenger demands. An irregular schedule that allows uneven headways can accommodate the dynamic demand patterns and accelerate the fleet utilization that is provided by a limited number of vehicles. This schedule type reduces the passenger waiting times and economizes the operation costs as well.

The transit scheduling problem has received much attention in the past years [1-3]. LeBlanc introduced a model for determining frequencies using a modal-split assignment programming model with distinct transit routes to capture the effects of increases or decreases on individual transit line frequencies [4]. Lee et al. revealed that the total fleet size required for the operation of a bus network with mixedsize vehicles is smaller than the fleet size necessary when all fleet vehicles have the same regular size [5]. Scheduling of urban transit network can be formulated as an optimization problem of minimizing the overall transfer time of transferring passengers and initial waiting time of the passengers waiting to board a vehicle at their point of origin [6]. The fleet size required for a bus line is decreased by inserting express and partial services in the schedule with alternative fleet assignment strategies, which take the headways as inputs [7]. De Palma and Lindsey examined the schedule delay 


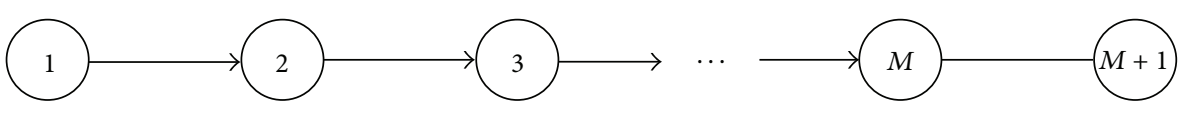

FIGURE 1: Illustration of an urban public transit line.

costs incurred from travelling earlier or later than desired and formulated an optimization model with the objective of minimizing the total riders' schedule delay costs [8]. Since passenger satisfaction with bus services has a high correlation with the attributes of the services supplied [9], Tom and Mohan formulated an optimization problem to minimize the bus operating cost and the passenger travel time by using frequency-coded genetic algorithms [10]. Assuming that no more than one passenger arrives at a station during a time interval, Niu and Zhou focused on optimizing a passenger train timetable in a heavily congested urban rail corridor [11].

A number of studies have somehow tried to model operational control strategies that are integrated with the scheduling problem to improve the efficiency of public transit systems. Site and Filippi proposed a particular service pattern including not only the short-turn strategy but also the vehicle size and frequencies that are required to supply the service over different operation periods [12]. By skipping a number of stops in high-frequency transit operations, the deadheading strategy could shorten the waiting time of passengers in the bus transport system [13]. Leiva et al. built an optimization method for designing limited-stop services, which minimizes both the travel time of passengers and the operating cost of an urban bus corridor by optimizing the transport services with different arrival frequencies of various types of vehicles [14]. Fu and Liu proposed a real-time scheduling strategy that aimed to strike an optimal balance between the benefits of operators and passengers [15]. Under the availability of real-time information and historical data of the system, the total user delay can be reduced by using a multipoint holding control strategy for a bus transit system [16]. Without considering the capacity constraints, Niu developed a nonlinear programming model and proposed a bilevel genetic algorithm to design a transit schedule with uneven headways and skip-stop operations [17].

The existing studies have paid much attention to regular schedules, but they have failed to explore the scheduling problem with uneven vehicle-departure headways based on time-dependent passenger demands. Adopting a similar approach to that used by dynamic traffic assignment in urban road network, our research divides an operation day into several equal time periods to formulate the transit scheduling problem. These periods cannot be too short; otherwise, obtaining the demand records will become difficult; however, if the period length is too long, the model loses the dynamicdemands feature. Under normal conditions, such as a oneminute period, we face a challenge that more than two passengers could arrive at a station simultaneously during one same period. Such a case can surely lead to some confusion when determining the queuing order for passengers arriving at this period under strict capacity constraints.
For a congested urban public transit line, some passengers must wait for an extended duration and then board the next or the third departure vehicles. When a vehicle departs from a station, under such time-dependent and congested conditions, we should decide which passengers can board the vehicle and which cannot be on the vehicle. This issue is also associated with the calculation of the number of boarded passengers for a departure vehicle at each station. The main aim of this research is to optimize the departure times of transit vehicles under strict capacity constraints. The model proposed in this paper is a biobjective programming problem, in which a novel heuristic algorithm is developed to solve the model.

The remainder of the paper is organized as follows. Section 2 presents an analysis of the vehicle fleet operations and the passenger travel behaviors, along with the presentation of the underlying assumptions. A biobjective optimization model for the scheduling problem with strict capacity constraints for an urban public transit line is given in Section 3. In Section 4, a heuristic algorithm based on intelligent search and local improvement is developed to solve the proposed model. In Section 5, a numerical example is provided to illustrate the application of the model and algorithm. The last section concludes the paper and outlines the possibilities for future research in related areas.

\section{Problem Statement}

2.1. Analysis. This paper focuses on an urban transit line which connects several residential areas and a workplace during the morning rush hours. The stations on the urban public transit line are numbered as $1,2, \ldots, M, M+1$ along the vehicle's forward direction, as shown in Figure 1, where $1,2, \ldots, M$ are the boarding stations; and $M+1$ is the destination station. In this scenario, the demand distribution is represented as multisource and single sink; specifically, the passengers board at the intermediate stations and reach their common destination station.

The passenger demands associated with urban public transit systems are characterized as being time dependent and stochastic; herewith, we use $[0, T]$ to index the study time horizon. In order to measure the dynamic demands, we divide the time horizon $[0, T]$ equally into several time periods (e.g., one minute as a period), and we use $\tau$ to denote a period and $\mathfrak{R}$ to represent the set of periods ( $\tau \in \mathfrak{R}$ ).

Taking into account that all passengers have the same destination, this study uses $P_{i}(\tau)$ to indicate the number of passengers who arrive at station $i(i=1,2, \ldots, M)$ during period $\tau(\tau \in \mathfrak{R})$ travelling to the destination station $M+1$. At the same time, this paper uses $\Omega$ to denote the set of vehicles that depart from the start station. Thus, the issue to be 
addressed in this paper is transformed into the determination of the departure time $d_{j}^{1}$ of transit vehicle $j(j \in \Omega)$ from station 1.

2.2. Assumptions. In order to objectively analyze the vehicle operation and the passengers' behaviour for urban public transit systems, the following assumptions are made throughout to facilitate the model formulation.

A1: all transit vehicles have the same capacity and follow strict capacity constraints. Once the number of in-vehicle passengers reaches the vehicle's full capacity, that vehicle is no longer available to any other passengers.

A2: all vehicles are scheduled according to the assumption that they travel at the same time between any two consecutive stations and they have the same dwell time for each station.

A3: the order for boarding a vehicle is randomly determined when several passengers arrive at a station simultaneously during the same period.

A4: all passengers who arrive at a station in different periods follow the principle of first-in-first-out (FIFO) when boarding a vehicle, which means that the passengers arriving within the earlier time periods board a vehicle prior to those arriving within the later periods.

A5: the total supply provided by the fleet meets the total passenger demand over the study horizon. Thus, some of the passengers may not board the current vehicle due to the oversaturation of demands, but the passengers waiting for the last vehicle could board the final run.

\section{Formulation}

3.1. Notation and Parameters. The following notation and parameters are used throughout this paper:

$i$ : index of stations, $i \in\{1,2, \ldots, M, M+1\}$;

$\mathfrak{R}$ : set of periods;

$\tau$ : index of periods, $\tau \in \mathfrak{R}$;

$\Omega$ : set of vehicles departing from the start station;

$j$ : index of vehicles, $j \in \Omega$;

$d_{j}^{i}$ : departure time of vehicle $j$ from station $i, i \in$ $\{1,2, \ldots, M\}, j \in \Omega$;

$P_{i}(\tau)$ : number of passengers who arrive at station $i$ during period $\tau$ and are travelling to the destination station, $i \in\{1,2, \ldots, M\}, \tau \in \mathfrak{R}$;

$Q_{j}^{i}$ : number of boarded passengers in vehicle $j$ during the vehicle's stay at station $i, j \in \Omega, i \in\{1,2, \ldots, M\}$;

$r^{i}$ : vehicle running time from station $i$ to station $i+1$, $i \in\{1,2, \ldots, M\}$;

$h^{i}:$ dwell time at station $i, i \in\{2,3, \ldots, M\}$; c: passenger loading capacity of a vehicle;

$I_{\text {min }}$ : prespecified minimum interval between two consecutive vehicles at the same station;

$I_{\text {max }}$ : prespecified maximum interval between two consecutive vehicles at the same station.

3.2. Constraints. The core decision variables are in fact the departure times for each vehicle at the start station. The detailed arrival and departure times at the other stations can be accordingly generated for scheduling applications. Given dwell time $h^{i}$ and running time $r^{i}$, the departure time for each vehicle at each station should satisfy the following equation:

$$
d_{j}^{i}=d_{j}^{i-1}+r^{i-1}+h^{i}
$$

Now, the minimum interval between two consecutive vehicles should be required to ensure the operational safety of vehicles, and the predetermined maximum interval should not be broken because the passenger waiting times at the stations cannot be too long. Thus, the departure times of two consecutive vehicles at the start station should satisfy the inequality

$$
I_{\min } \leq d_{j+1}^{1}-d_{j}^{1} \leq I_{\max }
$$

To accurately calculate the number of boarded passengers for a given vehicle at the intermediate stations, our research introduces two parameters, namely, the boarded period $b_{j}^{i}$ and residual number $s_{j}^{i}$. Figure 2 illustrates the two parameters by using the cumulative flow counts. The boarded period $b_{j}^{i}$ indicates a period in which at least one passenger arriving at station $i$ could board vehicle $j$, and no passenger at station $i$ can board vehicle $j$ offside this period. The residual number $s_{j}^{i}$ denotes the number of remaining passengers for vehicle $j$ at station $i$ during the boarded period $b_{j}^{i}$.

If we use $R_{i, j}(\tau)$ to indicate the number of boarded passengers in vehicle $j$ arriving at station $i$ during period $\tau$, the implication of a boarded period can be also expressed as follows:

$$
b_{j}^{i}=\max \left\{\tau \mid \tau \in \Re, R_{i, j}(\tau)>0\right\}
$$
follows:

The calculation of the residual number can be achieved as

$$
s_{j}^{i}=P_{i}\left(b_{j}^{i}\right)-R_{i, j}\left(b_{j}^{i}\right) .
$$




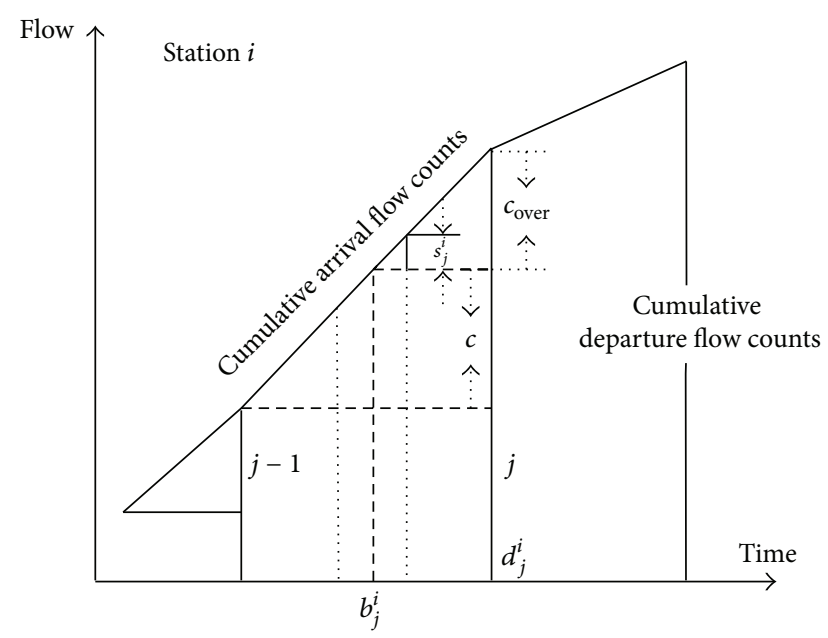

FIGURE 2: Illustration of board period and residual number.

On the other hand, the parameters of boarded period $b_{j}^{i}$ and residual number $s_{j}^{i}$ can be recursively calculated by

$$
\begin{aligned}
b_{j}^{i}=\min & \left\{\xi\left(d_{j}^{i}\right),\right. \\
& \left.\min \left\{h \mid \sum_{i^{\prime}=1}^{i-1} Q_{j}^{i^{\prime}}+s_{j-1}^{i}+\sum_{\tau \in\left(b_{j-1}^{i}, h\right]} P_{i}(\tau) \geq c\right\}\right\}, \\
s_{j}^{i} & =\max \left\{0, s_{j-1}^{i}+\sum_{\tau \in\left(b_{j-1}^{i}, b_{j}^{i}\right]} P_{i}(\tau)-c\right\},
\end{aligned}
$$

where $\xi\left(d_{j}^{i}\right)$ indicates a period which covers time point $d_{j}^{i}$ and that the boundary conditions are $b_{0}^{1}=0$ and $s_{0}^{1}=$ 0 . The number of boarded passengers in vehicle $j$ during the vehicle's stay at station $i, Q_{j}^{i}$, is thus determined by the equation

$$
Q_{j}^{i}=s_{j-1}^{i}+\sum_{\tau \in\left(b_{j-1}^{i}, b_{j}^{i}\right]} P_{i}(\tau)-s_{j}^{i}
$$

3.3. Objective. The transit operation is associated with the passengers and the bus company. The objective of the passengers is to minimize the overall waiting times at the stations, while the company wants to decrease the number of required vehicles. The first objective function, which aims to minimize the number of required vehicles, is

$$
\min |\Omega| .
$$

For a period with short length, this study assumes that when passengers arrive at a station during the same period, they actually all arrive at the start of that period. Let $\varphi(\tau)$ indicate the initial moment of period $\tau, \tau \in \Re$. When vehicle $j$ departs from station $i$, the total of the newly boarded passengers' waiting times is $\sum_{\tau \in\left(b_{j-1}^{i}, b_{j}^{i}\right]}\left(d_{j}^{i}-\varphi(\tau)\right) \times P_{i}(\tau)$, and the additional waiting time associated with all remaining passengers together is $\left(d_{j+1}^{i}-d_{j}^{i}\right) \times s_{j}^{i}$. As a result, the second objective function, which aims to minimize the total waiting time of all passengers at all stations, is formulated as follows:

$$
\begin{aligned}
& \min \sum_{i=1}^{M} \sum_{j \in \Omega}\left\{\left(d_{j+1}^{i}-d_{j}^{i}\right) \times s_{j}^{i}\right. \\
&\left.+\sum_{\tau \in\left(b_{j-1}^{i}, b_{j}^{i}\right]}\left(d_{j}^{i}-\varphi(\tau)\right) \times P_{i}(\tau)\right\} .
\end{aligned}
$$

\section{Algorithm}

The model proposed in this paper is a biobjective, nonlinear programming problem, which is difficult to solve with conventional, gradient-based methods or commercial optimization solvers. As a result, a heuristic algorithm based on intelligent search and local improvement is developed to solve the proposed model. This paper first assumes that the departure times of vehicles fall on integer minute points, such as 6:31 and 6:32. The main idea of the algorithm consists of two searches. Under the condition of the vehicles used in the least numbers, the biobjective is achieved by searching for the departure times in order to minimize the current cumulative waiting times, for each run at the start station.

At the start station, all possible time points are searched forward from the earliest time, moving ahead one minute at a time. The search process requires checking the predetermined vehicle-interval constraints and counting the total number of in-vehicle passengers. If the number of passengers in the vehicle reaches the capacity as the vehicle arrives at the destination station, the vehicle should depart at the current time point; otherwise, the search should move one unit forward to check the next point until the end of the time horizon. The objectives of minimizing the number of required vehicles and the total waiting time of passengers could be achieved by taking the obtained points as the departure times of vehicles at the start station. After the forward search, checking the length of the interval between the last two consecutive vehicles is required. The corresponding points should be adjusted by the backward search if the interval constraints are not satisfied.

4.1. Forward Search. With particular respect to the algorithm, this paper uses $p$ to indicate the search point associated with the departure time of the current vehicle, $A(j)$ to indicate the total number of boarded passengers for vehicle $j$ as it arrives at the destination station, and $H(j)=d_{j}^{1}-d_{j-1}^{1}$ to indicate the interval between vehicle $j$ and vehicle $j-1$ at the start station.

The search area of the first vehicle is $p \in\left[1, I_{\max }\right]$ during the forward process. Once the departure time $d_{j-1}^{1}$ of vehicle $j-1$ is determined, the search area of vehicle $j$ at the start station is $p \in\left[d_{j-1}^{1}+I_{\min }, d_{j-1}^{1}+I_{\max }\right]$ according to the 


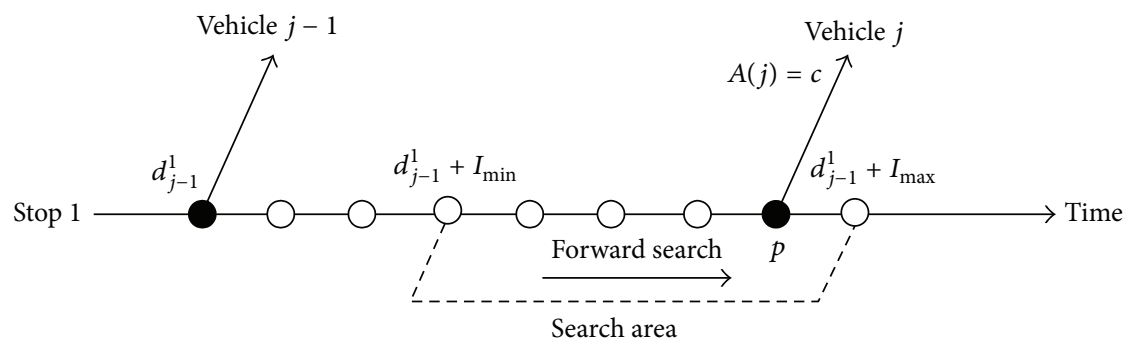

(a)

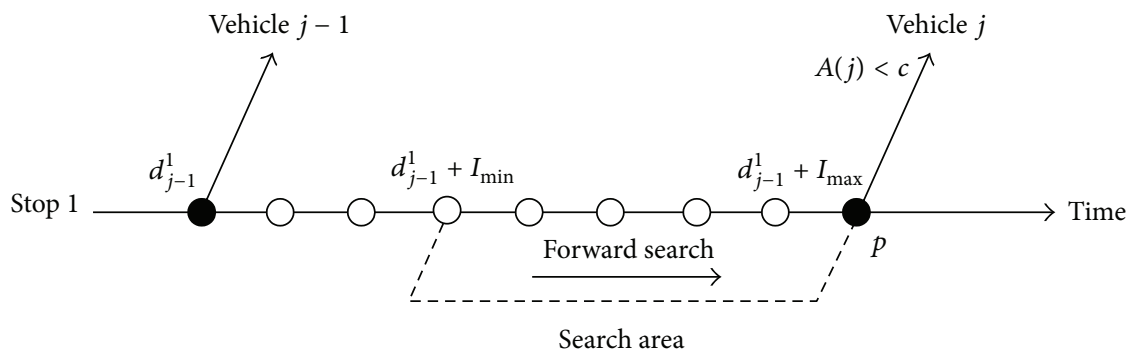

(b)

FIGURE 3: Illustration of search forward the possible times ahead one minute at a time.

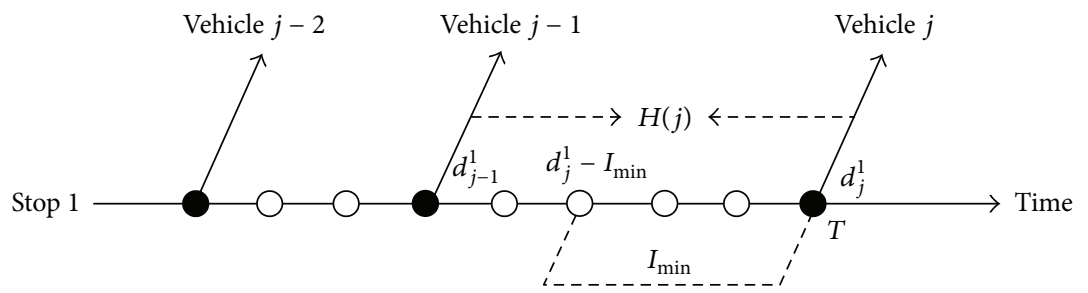

(a)

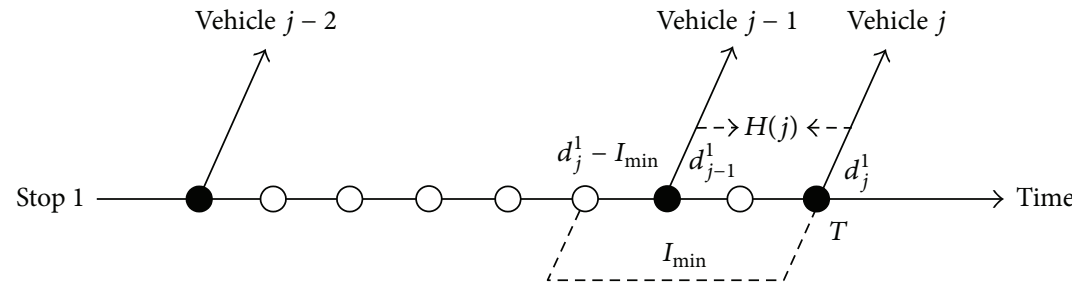

(b)

FIGURE 4: Checking the length of the interval between the last two consecutive vehicles.

headway constraints. All possible time points are searched forward from the start time $d_{j-1}^{1}+I_{\min }$ until a satisfactory time point is obtained. The following two cases are possible during the process of searching forward, as shown in Figure 3.

Figure 3(a) indicates that if the search reaches a point located within the predetermined area $\left[d_{j-1}^{1}+I_{\min }, d_{j-1}^{1}+\right.$ $\left.I_{\max }\right]$ and vehicle $j$ is already full (namely $A(j)=c$ ), the search should terminate and take the corresponding point as the departure time (namely $d_{j}^{1}=p$ ). Figure $3(\mathrm{~b}$ ) indicates that if the number of in-vehicle passengers $A(j)$ for vehicles $j$ departing from all the search points within the predetermined area is less than the capacity $c$, the end moment should be taken as the departure time (namely $d_{j}^{1}=$ $\left.d_{j-1}^{1}+I_{\max }\right)$.
4.2. Bound Check. According to assumption A5 that the provided supply meets the total passenger demand, the passengers waiting for the last vehicle can board the final run. To satisfy this assumption, this paper also assumes that the last vehicle departs from the start station at $T$, the study's last moment. When the forward search reaches the last moment, $T$, either all vehicles satisfy the headway constraints, or the interval between the last two vehicles does not satisfy the minimum headway condition. As a result, we need to perform the bound check while the departure times are generated from the forward search. During the bound check process, the following two cases, shown in Figure 4, are possible.

Figure 4(a) shows a case where the bound check is not required because the interval $H(j)$ between vehicle $j$ (the last 


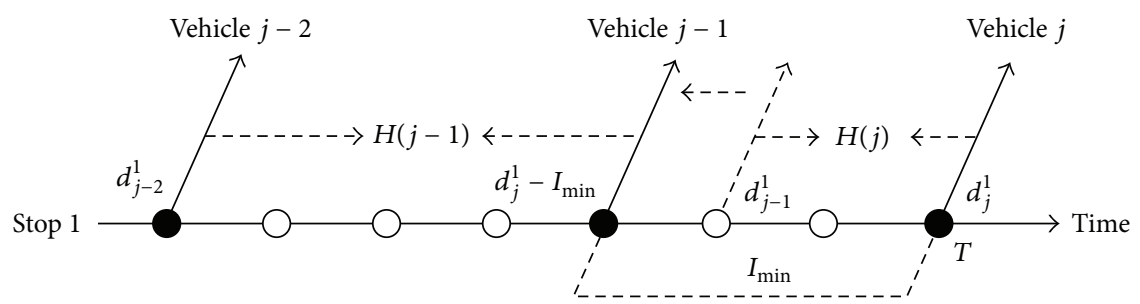

(a)

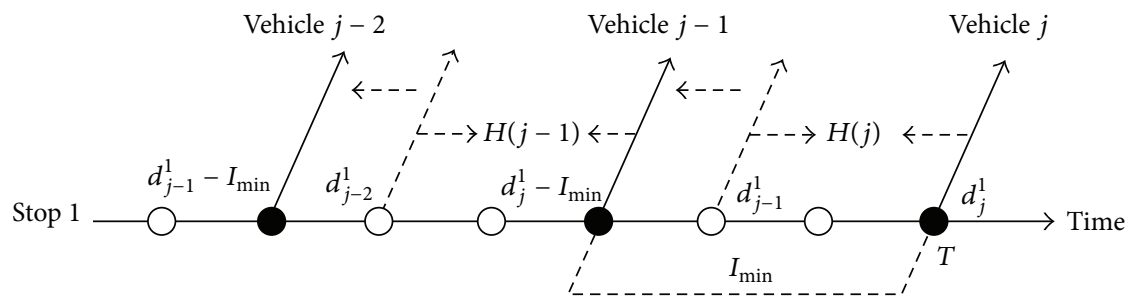

(b)

FIGURE 5: Illustration of backward adjustment in order to satisfy the minimum headway constraint.

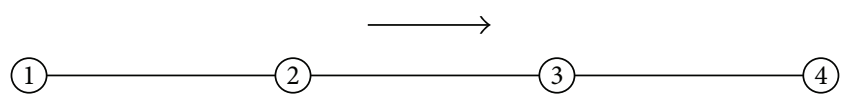

FIGURE 6: Illustration of a simple transit line.

run) and vehicle $j-1$ at the start station has already satisfied the minimum headway constraint. Figure 4(b) shows, however, a case in which the minimum headway constraint is not satisfied.

4.3. Backward Adjustment. In the case occurring in Figure 4(b), adjustments must be made to those departure times that do not satisfy the minimum headway constraint. The adjustment should be executed from the last vehicle by moving backward the associated time point when the vehicle-interval at the start station violates the headway constraint. The interval $H(j)$ between vehicle $j$ and vehicle $j-1$ at the start station must be checked, as well as the interval $H(j-1)$ between vehicle $j-1$ and vehicle $j-2$.

Figure 5(a) shows a case where the departure time of vehicle $j-1$ should be moved backward to a point at which the interval between vehicle $j$ and vehicle $j-1$ is $I_{\min }$ (so $\left.H(j)=I_{\min }\right)$, while the departure time of vehicle $j-2$ is not required to move backward because the new interval $H(j-1)$ between vehicle $j-1$ and vehicle $j-2$ still satisfies the minimum headway constraint. Figure 5(b), by contrast, shows a case where the departure time of vehicle $j-2$ is required to move backward after an adjustment is made to the departure time of vehicle $j-1$ because the new interval between vehicle $j-1$ and vehicle $j-2$ does not satisfy the minimum headway constraint.

4.4. Heuristic Algorithm. The above-described forward search, bound check, and backward adjustment form a heuristic algorithm, which is summarized as follows.
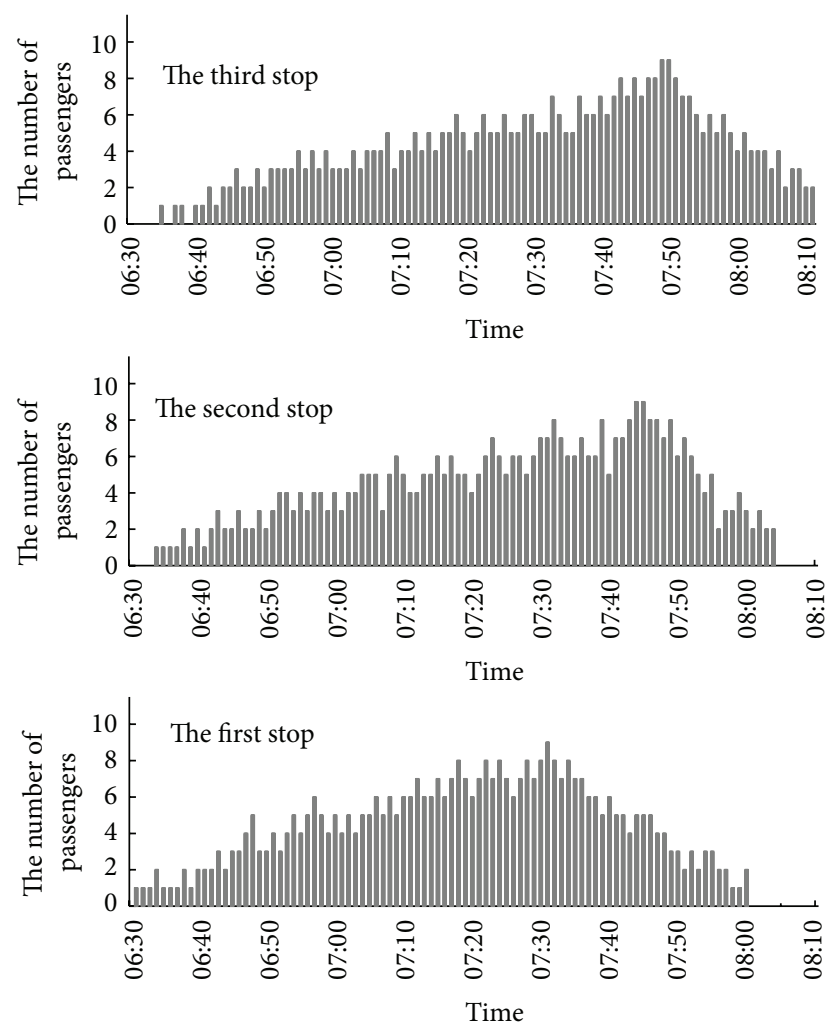

Figure 7: Passenger demands counted in one-minute intervals at intermediate stations.

Step 1. Set $p=1, j=1$, and $d_{0}^{1}=0$.

Step 2. Set $d_{j}^{1}=p$. If $d_{j}^{1} \geq T$, then $d_{j}^{1}=T$ and go to Step 5; otherwise, if $d_{j}^{1}-d_{j-1}^{1}=I_{\max }$, go to Step 4 , and if $d_{j}^{1}-d_{j-1}^{1}<$ $I_{\text {max }}$, go to Step 3 . 
TABLE 1: The departure times and the associated numbers of boarded and in-vehicle passengers.

\begin{tabular}{|c|c|c|c|c|c|c|c|c|c|c|}
\hline \multirow{2}{*}{$\begin{array}{l}\text { Vehicle } \\
\text { number }\end{array}$} & \multirow{2}{*}{$\begin{array}{l}\text { Departure } \\
\text { time }\end{array}$} & \multicolumn{3}{|c|}{ The first station } & \multicolumn{3}{|c|}{ The second station } & \multicolumn{3}{|c|}{ The third station } \\
\hline & & $\mathrm{BP} / \mathrm{RN}$ & $\begin{array}{c}\text { Boarded } \\
\text { passengers }\end{array}$ & $\begin{array}{l}\text { In-vehicle } \\
\text { passengers }\end{array}$ & $\mathrm{BP} / \mathrm{RN}$ & $\begin{array}{c}\text { Boarded } \\
\text { passengers }\end{array}$ & $\begin{array}{l}\text { In-vehicle } \\
\text { passengers }\end{array}$ & $\mathrm{BP} / \mathrm{RN}$ & $\begin{array}{c}\text { Boarded } \\
\text { passengers }\end{array}$ & $\begin{array}{l}\text { In-vehicle } \\
\text { passengers }\end{array}$ \\
\hline 1 & $6: 40$ & $10 / 0$ & 13 & 13 & $15 / 0$ & 17 & 30 & $22 / 0$ & 26 & 56 \\
\hline 2 & $6: 47$ & $17 / 0$ & 19 & 19 & $22 / 0$ & 19 & 38 & $29 / 2$ & 22 & 60 \\
\hline 3 & $6: 53$ & $23 / 0$ & 22 & 22 & $28 / 0$ & 22 & 44 & $34 / 6$ & 16 & 60 \\
\hline 4 & $6: 58$ & $28 / 0$ & 25 & 25 & $33 / 0$ & 18 & 43 & $38 / 9$ & 17 & 60 \\
\hline 5 & $7: 02$ & $32 / 0$ & 18 & 18 & $37 / 0$ & 18 & 36 & $44 / 3$ & 24 & 60 \\
\hline 6 & $7: 06$ & $36 / 0$ & 20 & 20 & $41 / 0$ & 20 & 40 & $48 / 3$ & 20 & 60 \\
\hline 7 & $7: 10$ & $40 / 0$ & 22 & 22 & $45 / 0$ & 20 & 42 & $52 / 5$ & 18 & 60 \\
\hline 8 & $7: 14$ & $44 / 0$ & 25 & 25 & $49 / 0$ & 21 & 46 & $54 / 12$ & 14 & 60 \\
\hline 9 & $7: 17$ & $47 / 0$ & 20 & 20 & $52 / 0$ & 15 & 35 & $59 / 4$ & 25 & 60 \\
\hline 10 & $7: 20$ & $50 / 0$ & 21 & 21 & $55 / 0$ & 18 & 39 & $62 / 0$ & 21 & 60 \\
\hline 11 & $7: 24$ & $54 / 0$ & 30 & 30 & $59 / 0$ & 23 & 53 & $64 / 16$ & 7 & 60 \\
\hline 12 & $7: 27$ & $57 / 0$ & 20 & 20 & $62 / 0$ & 22 & 42 & $67 / 17$ & 18 & 60 \\
\hline 13 & $7: 30$ & $60 / 0$ & 23 & 23 & $65 / 0$ & 19 & 42 & $70 / 20$ & 18 & 60 \\
\hline 14 & $7: 32$ & $62 / 0$ & 17 & 17 & $67 / 0$ & 13 & 30 & $74 / 5$ & 30 & 60 \\
\hline 15 & $7: 35$ & $65 / 0$ & 22 & 22 & $70 / 0$ & 19 & 41 & $76 / 9$ & 19 & 60 \\
\hline 16 & $7: 38$ & $68 / 0$ & 19 & 19 & $73 / 0$ & 22 & 41 & $79 / 16$ & 19 & 60 \\
\hline 17 & $7: 41$ & $71 / 0$ & 16 & 16 & $76 / 0$ & 26 & 42 & $81 / 18$ & 18 & 60 \\
\hline 18 & $7: 44$ & $74 / 0$ & 14 & 14 & $79 / 0$ & 23 & 37 & $84 / 11$ & 23 & 60 \\
\hline 19 & $7: 48$ & $78 / 0$ & 18 & 18 & $83 / 0$ & 24 & 42 & $88 / 13$ & 18 & 60 \\
\hline 20 & $7: 53$ & $83 / 0$ & 13 & 13 & $88 / 0$ & 17 & 30 & $95 / 2$ & 30 & 60 \\
\hline 21 & $8: 00$ & $90 / 0$ & 14 & 14 & $95 / 0$ & 16 & 30 & $102 / 0$ & 14 & 44 \\
\hline
\end{tabular}

TABLE 2: The departure times of vehicles at start station associated with a regular schedule.

\begin{tabular}{|c|c|c|c|c|c|}
\hline Vehicle number & Departure time & Vehicle number & Departure time & Vehicle number & Departure time \\
\hline 1 & $6: 36$ & 8 & 7:08 & 15 & $7: 36$ \\
\hline 2 & $6: 42$ & 9 & $7: 12$ & 16 & $7: 40$ \\
\hline 3 & $6: 48$ & 10 & $7: 16$ & 17 & $7: 44$ \\
\hline 4 & $6: 52$ & 11 & $7: 20$ & 18 & $7: 48$ \\
\hline 5 & $6: 56$ & 12 & $7: 24$ & 19 & $7: 52$ \\
\hline 6 & 7:00 & 13 & $7: 28$ & 20 & $7: 56$ \\
\hline 7 & $7: 04$ & 14 & $7: 32$ & 21 & $8: 00$ \\
\hline
\end{tabular}

Step 3. Calculate $A(j)=\sum_{s=1}^{M} Q_{j}^{s}$. If $A(j)=c$, go to Step 4; otherwise, set $p=p+1$ and go to Step 2 .

Step 4. Set $j=j+1, p=p+I_{\min }$, and go to Step 2 .

Step 5. Calculate $H(j)=d_{j}^{1}-d_{j-1}^{1}$.

Step 6. If $H(j) \geq I_{\min }$, set $H(j-1)=d_{j-1}^{1}-d_{j-2}^{1}$ and go to Step 7; otherwise, set $d_{j-1}^{1}=d_{j-1}^{1}-1$ and go to Step 5 .

Step 7. If $H(j-1) \geq I_{\min }$, then stop; otherwise, set $j=j-1$ and go to Step 5.

\section{Numerical Example}

In this section, we consider a simple transit line on which there are three boarding stations and one destination station, as shown in Figure 6. The running time from station 1 to station 2 is 4 minutes; from station 2 to station 3 , the time is 6 minutes; and from station 3 to station 4 , the time is 15 minutes. The dwell time at each station is 1 minute. The study horizon is from 6:30 to 8:00, and the length of each subdivision is 1 minute. Each transit vehicle has a capacity of 60 passengers, and the maximum and minimum headways are 10 minutes and 2 minutes, respectively. 
TABLE 3: Comparison of regular schedule with irregular schedule.

\begin{tabular}{lccccc}
\hline Schedule & $\begin{array}{c}\text { Number of departed } \\
\text { vehicles }\end{array}$ & $\begin{array}{c}\text { Total waiting time } \\
(\mathrm{min})\end{array}$ & $\begin{array}{c}\text { Average waiting } \\
\text { time }(\mathrm{min})\end{array}$ & $\begin{array}{c}\text { Boarded passenger } \\
(\%)\end{array}$ & $\begin{array}{c}\text { Average load rate } \\
(\%)\end{array}$ \\
\hline Irregular & 21 & 1417 & 1.14 & 1240 & 98.41 \\
Regular & 21 & 3943 & 3.38 & 1168 & 92.70 \\
\hline
\end{tabular}

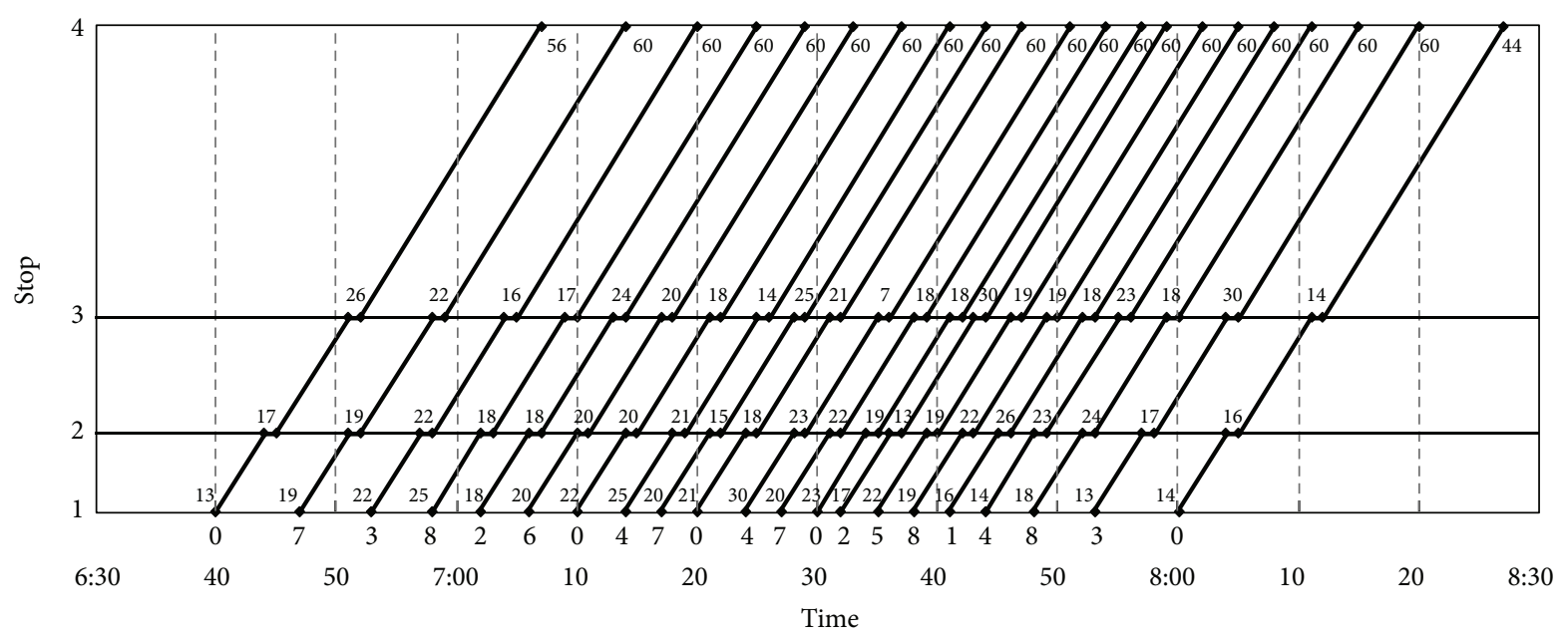

FIGURE 8: Illustration of transit schedule corresponding to heuristic algorithm.

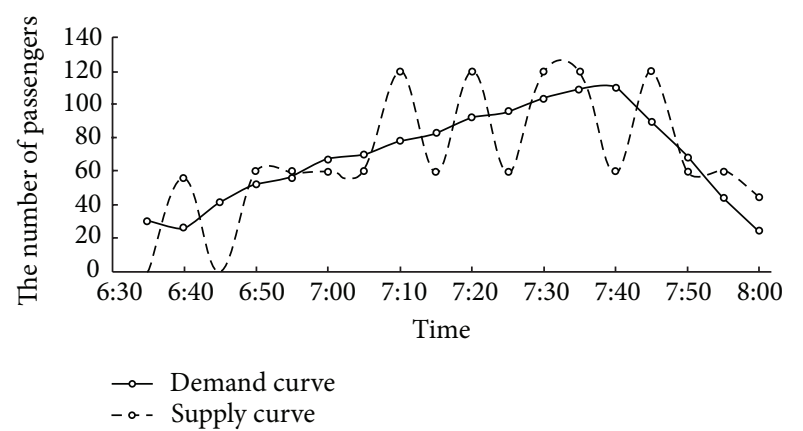

FIGURE 9: Illustration of demand and supply curves.

The period-dependent passenger demands, as shown in Figure 7, are illustrated by the total number of passengers arriving at the intermediate stations within each minute. By using the heuristic algorithm developed above, the departure times of vehicles at the start station and the associated numbers of boarded and in-vehicle passengers are determined; the results are given in Table 1 . In this case, the necessary number of vehicles is 21 .

In Table $1, \mathrm{BP}$ and $\mathrm{RN}$ denote board period and residual number, respectively, and the total waiting time of all passengers at the stations is 1417 minutes. Using the information presented in Table 1, the corresponding schedule was created; this schedule is shown in Figure 8. Figure 8 shows that longer intervals between departure times occur from 6:30 to 7:00 than from 7:30 to 7:45. This temporal distribution of vehicles is approximately consistent with the passenger demands.

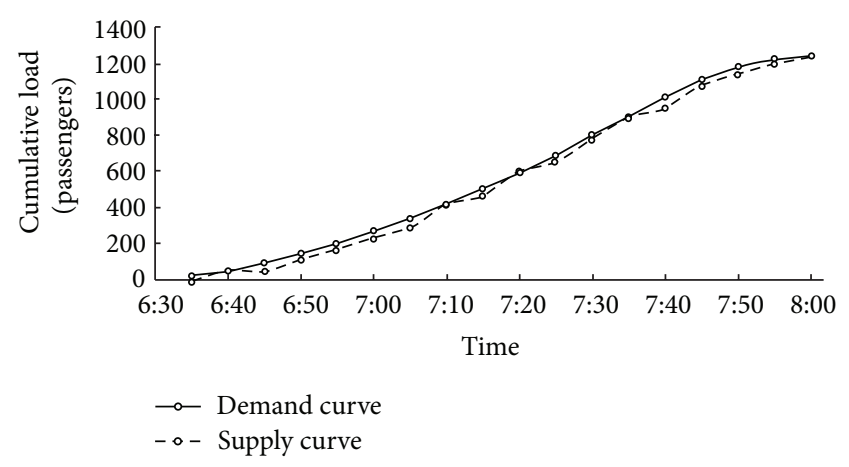

FIGURE 10: Cumulative curves of the demand and supply.

In Figure 9, the two curves illustrate the relation between the demand and supply associated with the designed schedule for the transit line. The demand curve shows the total number of passengers waiting at the stations every five minutes. The five-minute periods used at a subsequent station should be equivalent to those at the first station delayed by the running time between this subsequent station and the first station because the same vehicle arrives at that subsequent station after no less than the running time between the stations. The supply curve displays the number of boarded passengers every five minutes as the vehicles follow the designed schedule. The corresponding cumulative curves are shown in Figure 10, which indicates the correspondence between the demand and supply.

In order to further demonstrate the advantage of the method proposed in this paper, Table 2 shows a regular 
schedule associated with the same fleet supply and other parameters; in this case, 72 passengers cannot board the last vehicle.

In Table 2, the intervals between the first three vehicles are 6 minutes, and those between the remaining vehicles are 4 minutes. A comparison between the designed schedule and the regular schedule is made in Table 3.

From Table 3, we can see that all passengers can board the vehicles using the designed schedule, while some cannot when using the regular one. Moreover, the average waiting time associated with the proposed method is reduced by $66.27 \%$, which further demonstrates the efficiency of the method proposed in this paper.

\section{Conclusions}

This paper studies the transit scheduling problem to optimize the departure times of vehicles for a congested urban public transit line. Based on some reasonable assumptions, a biobjective optimization model has been established to minimize the number of required vehicles and the total waiting times of passengers. Under period-dependent demand and strict capacity conditions, our method has fully considered the situation in which there are more than two passengers arriving at a station simultaneously during one same period.

The proposed model is a nonlinear programming problem, which is difficult to solve with a conventional approach. Due to the potential of heuristic approaches to handle complex problems, a hybrid procedure embedded in a heuristic algorithm is applied to solve the model. The validation of the model and the algorithm has been successfully tested with the help of a numerical example. In future research, we will consider the response of passengers to the optimized schedule and extend our method to a more general transit case.

\section{Acknowledgment}

The work described in this paper was supported by the National Natural Science Foundation of China (Grant no. 71261014).

\section{References}

[1] S. C. Wirasinghe, "Initial planning for urban transit systems," in Advanced Modeling for Transit Operations and Service Planning, pp. 1-29, Elsevier, Oxford, UK, 2003.

[2] A. Ceder, Public Transit Planning and Operation, Elsevier, Linacre House, Jordan Hill, Oxford, UK, 2007.

[3] V. Guihaire and J.-K. Hao, "Transit network design and scheduling: a global review," Transportation Research Part A, vol. 42, no. 10, pp. 1251-1273, 2008.

[4] L. J. LeBlanc, “Transit system network design," Transportation Research Part B, vol. 22, no. 5, pp. 383-390, 1988.

[5] K. K.-T. Lee, S. H. F. Kuo, and P. M. Schonfeld, "Optimal mixed bus fleet for urban operations," Transportation Research Record, no. 1503, pp. 39-48, 1995.

[6] P. Chakroborty, K. Deb, and P. S. Subrahmanyam, "Optimal scheduling of urban transit systems using genetic algorithms,"
Journal of Transportation Engineering, vol. 121, no. 6, pp. 544$553,1995$.

[7] T. A. S. Vijayaraghavan and K. M. Anantharamaiah, "Fleet assignment strategies in urban transportation using express and partial services," Transportation Research Part A, vol. 29, no. 2, pp. 157-171, 1995.

[8] A. De Palma and R. Lindsey, "Optimal timetables for public transportation," Transportation Research Part B, vol. 35, no. 8, pp. 789-813, 2001.

[9] P. Prioni and D. Hensher, "Measuring service quality in scheduled bus services," Journal of Public Transportation, vol. 3, no. 2, pp. 51-74, 2000.

[10] V. M. Tom and S. Mohan, "Transit route network design using frequency coded genetic algorithm," Journal of Transportation Engineering, vol. 129, no. 2, pp. 186-195, 2003.

[11] H. M. Niu and X. Zhou, "Optimizing urban rail timetable under time-dependent demand and oversaturated conditions," Transportation Research Part C, vol. 36, pp. 212-230, 2013.

[12] P. D. Site and F. Filippi, "Service optimization for bus corridors with short-turn strategies and variable vehicle size," Transportation Research Part A, vol. 32, no. 1, pp. 19-38, 1998.

[13] X. J. Eberlein, N. H. M. Wilson, C. Barnhart, and D. Bernstein, "The real-time deadheading problem in transit operations control," Transportation Research Part B, vol. 32, no. 2, pp. 77100, 1998.

[14] C. Leiva, J. C. Muñoz, R. Giesen, and H. Larrain, "Design of limited-stop services for an urban bus corridor with capacity constraints," Transportation Research Part B, vol. 44, no. 10, pp. 1186-1201, 2010.

[15] L. Fu and Q. Liu, "Real-time optimization model for dynamic scheduling of transit operations," Transportation Research Record, no. 1857, pp. 48-55, 2003.

[16] L. A. Koehler, W. Kraus Jr., and E. Camponogara, "Iterative quadratic optimization for the bus holding control problem," IEEE Transactions on Intelligent Transportation Systems, vol. 12, no. 4, pp. 1568-1575, 2011.

[17] H. M. Niu, "Determination of the skip-stop scheduling for a congested transit line by bilevel genetic algorithm," International Journal of Computational Intelligence Systems, vol. 4, no. 6, pp. 1158-1167, 2011. 


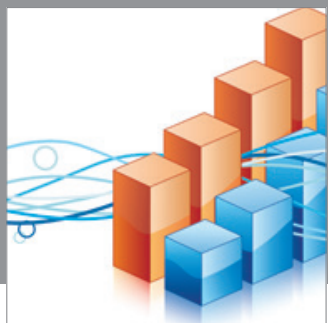

Advances in

Operations Research

mansans

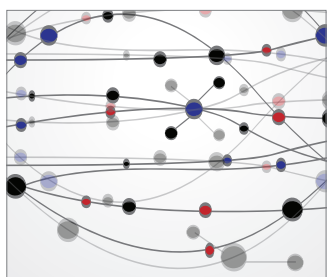

The Scientific World Journal
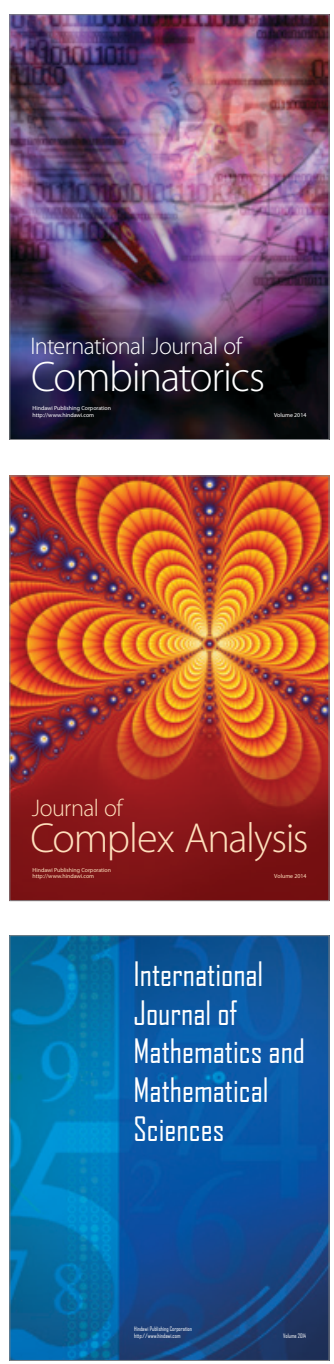
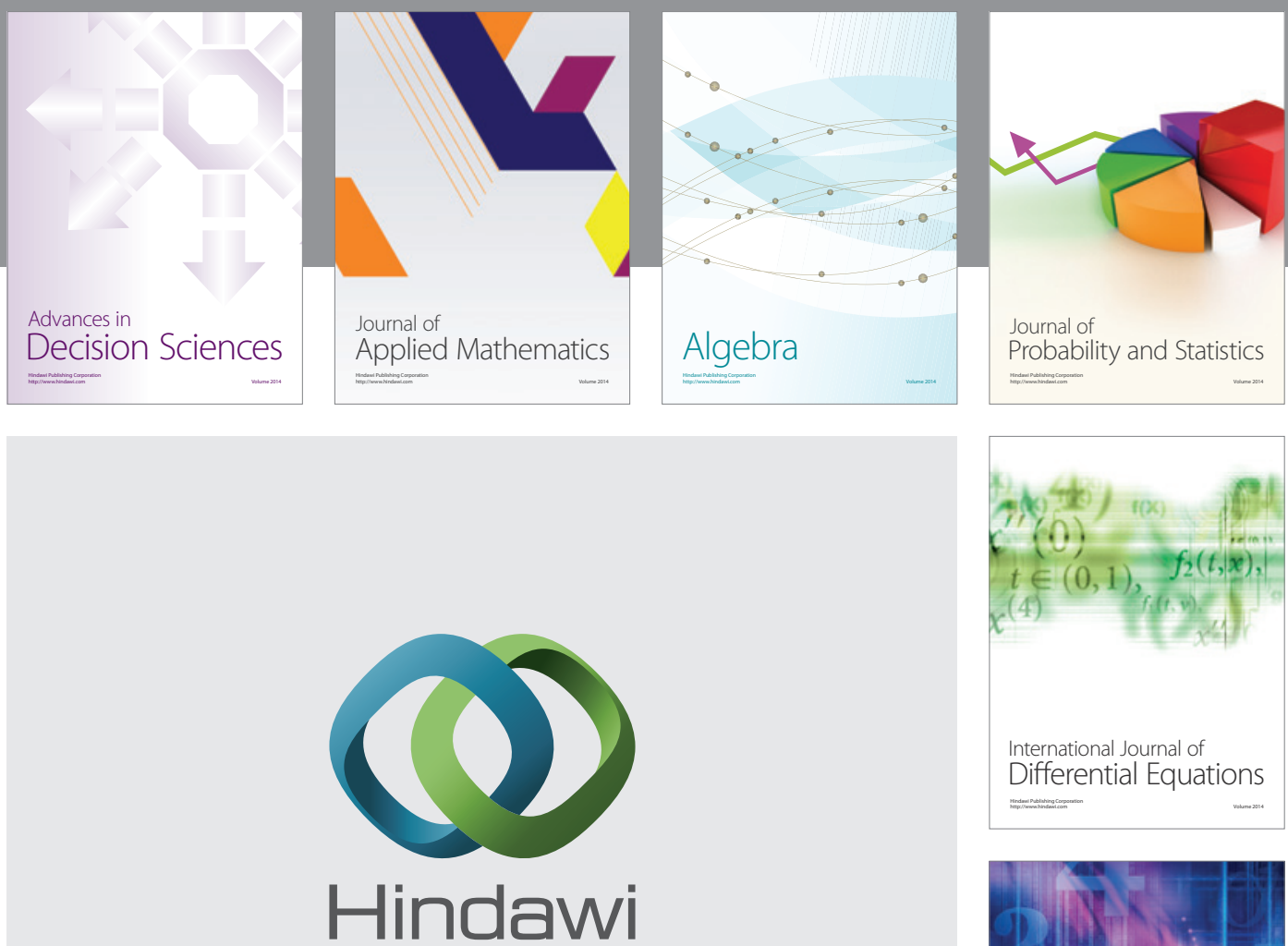

Submit your manuscripts at http://www.hindawi.com
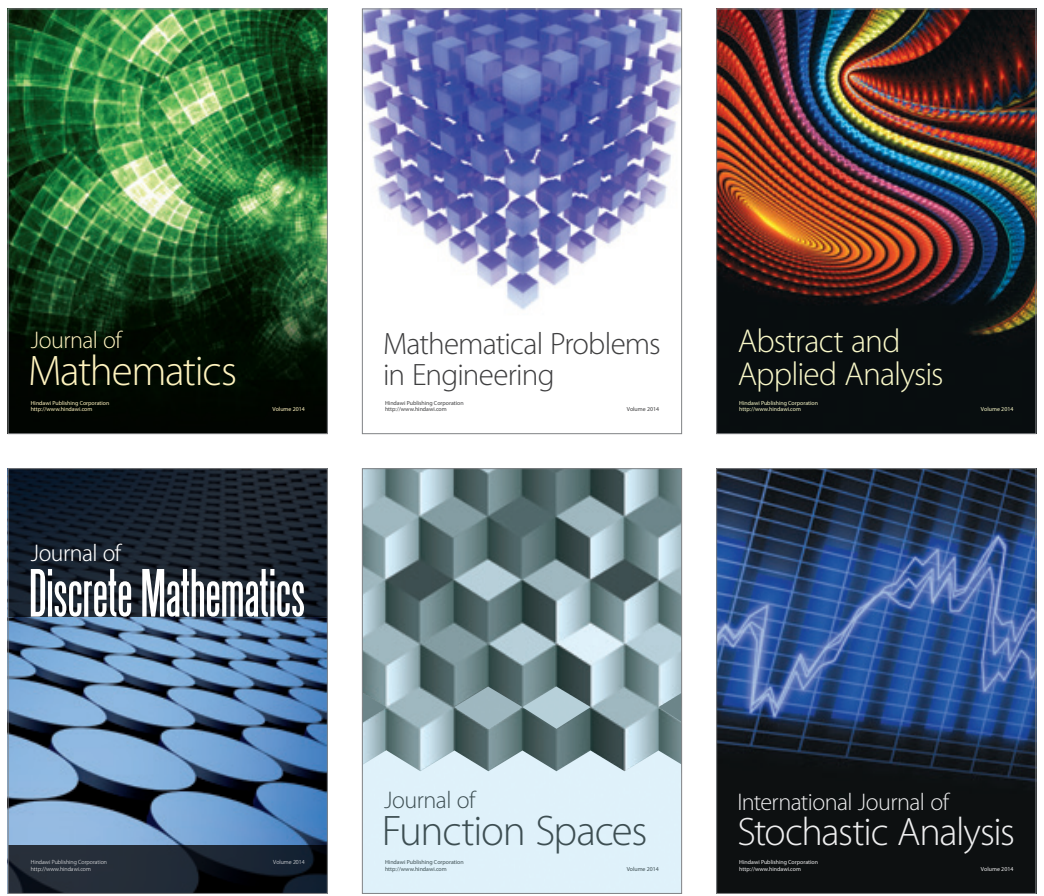

Journal of

Function Spaces

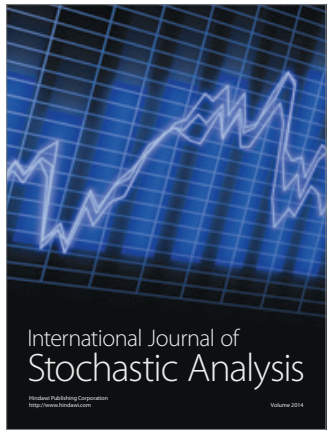

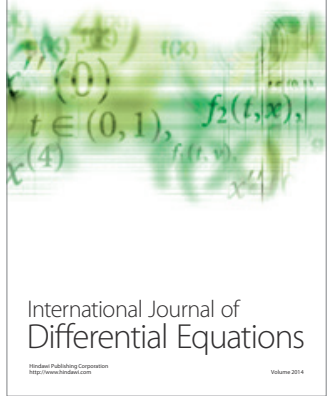
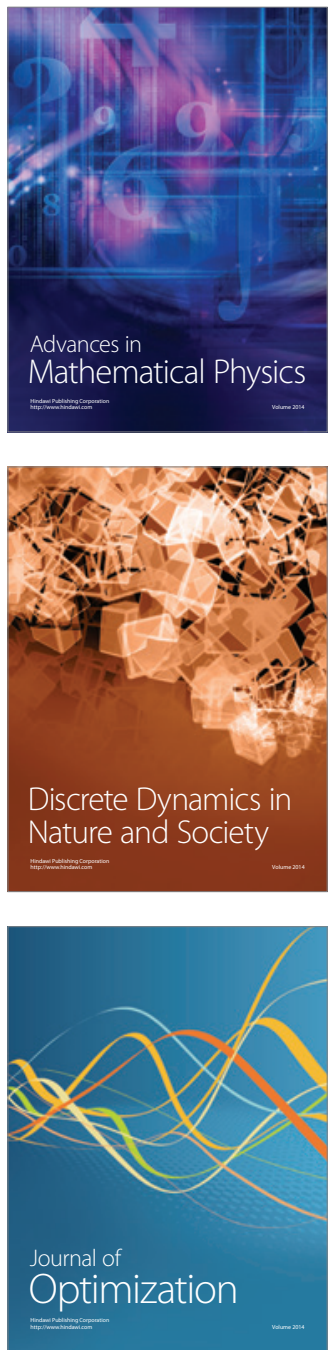